PHYSICAL REVIEW E 73, 065602(R) (2006)

\title{
Reduced transport velocity of multiply scattered light due to resonant scattering
}

\author{
Martin Störzer, Christof M. Aegerter, and Georg Maret \\ Fachbereich Physik, University of Konstanz, Universitätstrasse 10, 78457 Konstanz, Germany
}

(Received 2 March 2006; published 9 June 2006)

\begin{abstract}
The transport properties of photons traveling through random media are of great fundamental and applied importance. For instance the dwell time due to resonant Mie scattering can lead to a significant reduction in transport velocity. Here, we have measured directly the energy-transport velocity of photons in strongly scattering media using a combination of time resolved transmission, measuring the diffusion coefficient, and angular resolved backscattering, yielding the transport mean free path. We find that the transport velocity is strongly reduced when the effective diameter of the scattering particles is a multiple of half the wavelength. This is consistent with the occurrence of resonant Mie scattering in the samples. We compare our data to previous theoretical calculations of the transport velocity as a function of scatterer size.
\end{abstract}

DOI: 10.1103/PhysRevE.73.065602

PACS number(s): 42.25.Dd, 05.60.-k, 42.25.Bs

Fifty years ago, Wigner calculated the properties of a quantum particle captured in a potential resonant to its wavelength [1]. He obtained that the resonant scattering of such a particle on the potential leads to an increased dwell time. In recent years, this has been implemented, using photons as the scattering particle, in many different experimental circumstances. Using the scattering properties of cold atomic gases, it has, for instance, been possible to slow down light transport by many orders of magnitude [2]. On the other hand, using properly tuned microspheres, it has been possible to create optical cavities with $Q$ factors in excess of $10^{10}$ [3], which in turn has had many applications in photonics [4] as well as biosensing [5]. Furthermore, the resonant increase of intensity is important in the production of photonic crystals $[6,7]$, where band gaps of light transport appear.

In nature however, multiple scattering is dominating most effects, as can, for instance, be seen from the fact that most materials appear to be turbid. Photons carry out a random walk through the material, characterized by a transport mean free path $l^{*}$ and a diffusion coefficient $D=v l^{*} / 3$. Due to the wave-nature of photons, the resonant scattering induced dwell time induces a reduction in the transport velocity $v_{T}$, even in a multiple scattering process [8]. On the other hand, resonant Mie scattering also leads to an increase of the scattering cross section of the particles and hence to a decrease of $l^{*}$ in multiple scattering samples. At small values of $l^{*}$, interference effects start to influence the multiple scattering transport behavior as well. Constructive interference on counterpropagating paths for instance leads to enhanced backscattering, known as weak localization $[9,10]$. At even higher turbidity, when $l^{*}$, multiplied by the wave number, $k$, approaches unity [11], transport is exponentially suppressed [12] and photons are observed to remain inside a multiple scattering sample for times longer than expected from a diffusive picture [13]. This is due to a renormalization of the diffusion coefficient [14], which in this context is known as Anderson, or strong, localization of light $[6,12,15]$.

Due to the fact that both a reduction in $v_{T}$ and $l^{*}$ lead to a reduction in transport indicated by a delay in photons traveling through the sample, a separation of localization and resonance scattering can be difficult. Early measurements of $D$, which have found anomalously low values, have for instance been interpreted as the onset of Anderson localization [16].
However, theoretical calculations of $v_{T}$ corresponding to comparable multiple scattering media and explicitly taking into account resonant scattering [8] have shown that $v_{T}$ decreases by a factor of up to 7 for certain effective particle diameters. Thus a small value of $D$ was more likely due to a reduction of the transport velocity $[8,17]$ and does not necessarily imply a small value of $l^{*}$. In fact van Albada et al. [8] have shown experimentally for a single sample that such a reduction can indeed be observed. However, a clear demonstration of the resonant nature, i.e., the increased reduction of $v_{T}$ at specific particle sizes or wavelengths is still lacking. In addition, when one tries to maximize the turbidity in the approach to the Ioffe-Regel criterion [11], the particle sizes necessarily become of the order of the wavelength of light. Therefore one needs to be able to clearly separate the effects of resonant scattering and localization when investigating deviations from classical diffusion in the context of Anderson localization of light [13].

Here, we directly measure $v_{T}$ for many different particle sizes in order to study the effect of resonant scattering. To achieve this, both $D$ and $l^{*}$ have to be quantified independently. From time resolved transmission [16,19], we obtain the diffusion coefficient, while the value of $l^{*}$ is obtained from the angular dependence of the reflection from the same sample $[9,10]$. We can then eliminate the mean free path $l^{*}$ from the diffusion coefficient which leaves us with a direct measure of $v_{T}$.

To measure $D$, a single photon counting method $[16,19]$ is used to obtain time of flight (TOF) distributions of diffusing photons. Here the broadening of a picosecond light pulse traveling through a multiple scattering sample is recorded by taking a histogram of many single photon counting measurements. Our setup consists of a Rhodamin6G dye laser pumped by a mode locked $\mathrm{Ar}^{+}$laser issued with a cavity dumper, producing pulses with a width of $\sim 20$ ps at a wavelength of $590 \mathrm{~nm}$. The measured TOFs were corrected with the pulse shape recorded without the sample to get rid of an artificial broadening of the signal. This is done by deconvoluting the TOF signal and the reference pulse shape in Fourier space [13]. As samples, we use powders of ground $\mathrm{TiO}_{2}$ in its rutile structure. Such powders are commercially available as pigments for white paint with varying particle sizes, e.g., from Aldrich (Ti-pure) and DuPont (R101, R104, R700, 


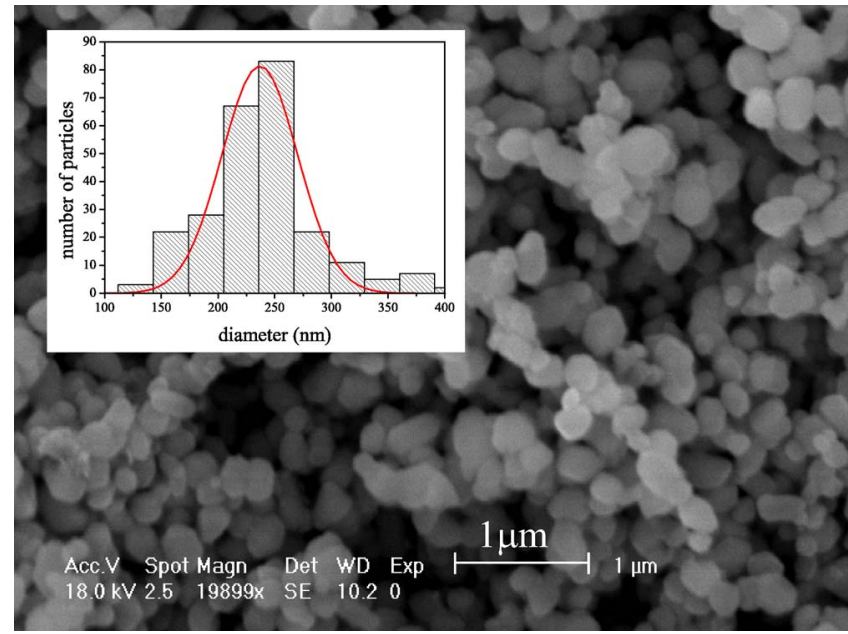

FIG. 1. (Color online) An electron micrograph of sample R706 used for characterization of the particle size distribution. The corresponding histogram of particle sizes is shown in the inset, which shows that these particles have an average diameter of $230 \mathrm{~nm}$ with a polydispersity of $15 \%$. Thus they are in a region where resonant scattering may be expected and where narrow resonances may be resolved.

R706, R900, and R902). The rutile structure of $\mathrm{TiO}_{2}$ has a refractive index of up to 2.8 at a wavelength of $590 \mathrm{~nm}$ and the ground particles have diameters ranging from 220 to $540 \mathrm{~nm}$ with a polydispersity of about $20 \%$. The particle sizes were characterized from scanning electron micrographs like the one shown in Fig. 1 for sample R706. The inset of the figure shows a histogram of particle diameters corresponding to the electron micrograph. As can be seen, the particle sizes are of the order of the wavelength of light, with a reasonably low polydispersity, such that effects of resonant scattering may be observed. In order to get a sufficiently turbid sample the powders were compressed to a filling fraction of $\sim 40 \%$.

Figure 2 shows such a time resolved transmission measurement. From diffusion theory, the pulse shape of such a time of fight measurement can be calculated analytically to be $[16,18]$

$$
T(t) \propto e^{-c t / l_{a}} \sum_{n}(-1)^{n} n^{2} e^{-n^{2} \pi^{2} D t / L^{2}},
$$

where $L$ is the thickness of the sample and $l_{a}$ is the absorption length. The dotted curve corresponds to the theoretical fit using diffusion theory where the diffusion coefficient and the absorption length were put in as fit parameters. There is only little covariance in the two fit parameters, as the diffusion coefficient is dominant for small times (around the maximum), whereas absorption is the main contribution in the long time limit. Typical values of the measured diffusion coefficients range from 10 to $30 \mathrm{~m}^{2} / \mathrm{s}$ with absorption lengths between 0.3 and $2.6 \mathrm{~m}$, depending on the sample.

In order to measure the transport mean free path $l^{*}$ we use a custom designed setup [20] to measure the coherent backscattering cone. Our setup consists of 256 photodiodes attached to an arc with a radius of $0.6 \mathrm{~m}$ in order to get a

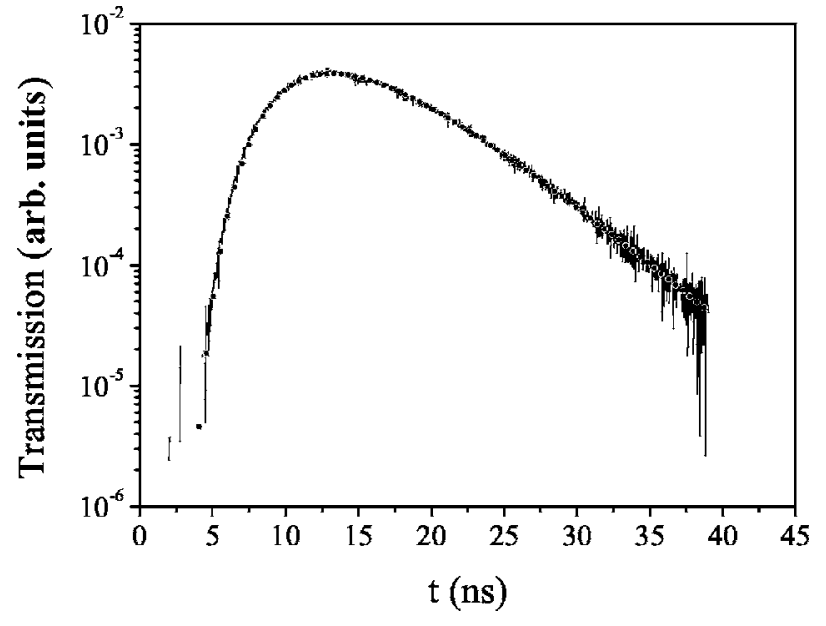

FIG. 2. Time resolved transmission through the Aldrich $\mathrm{TiO}_{2}$ sample consisting of particles with a mean diameter of $540 \mathrm{~nm}$ and a polydispersity of $25 \%$. The dotted curve represents a diffusion fit including absorbtion according to Eq. (1) to the measured data. The peak position as well as the exponential slope allow a determination of the diffusion coefficient as $D=22 \mathrm{~m}^{2} / \mathrm{s}$ as well as the absorption length $l_{a}=2.6 \mathrm{~m}$ for this sample given the thickness of the sample, which was $L=2.21 \mathrm{~mm}$.

sufficient angular resolution. Here, the resolution is $\sim 1^{\circ}$ for backscattering angles $|\theta|>10^{\circ}$ and $0.14^{\circ}$ for $|\theta|<10^{\circ}$. In addition, the central part of the backscattering cone, $|\theta|<3^{\circ}$ was measured separately using a beamsplitter and a charged coupled device camera to a resolution of $0.02^{\circ}$ in order to be able to resolve the tip of the cone at $0^{\circ}$. The measurements were done using circularly polarized light in order to reduce the influence of singly scattered light. A combination of those two methods results in high resolution coherent backscattering signals up to an angle of $65^{\circ}$. This is shown in Fig. 3 for the same sample characterized by TOF transmission in Fig. 2. From the full width at half maximum of the angular resolved backscattering signal, $k l^{*}$ can be determined directly via $\mathrm{FWHM}=0.954\left(k l^{*}\right)^{-1}[21]$. Due to a jump in the refractive index at the boundary, internal reflections may occur that lead to a narrowing of the backscattering signal [22]. To correct for this, we have calculated the effective refractive index of the medium using the energy coherent potential approximation (ECPA) $[23,24]$ for our samples in order to determine the reflectivities. It is then possible to correct for the narrowing and obtain reliable estimates of $l^{*}$ [22].

Thus, knowing the diffusion coefficient $D=v_{T} l^{*} / 3$ and the transport mean free path $l^{*}$ we are able to calculate $v_{T}$ directly. Repeating the presented measurements for different effective particle sizes $d / \lambda$ both with different particles, thus changing $d$, as well as with different wavelengths, one gets a dependance of the relative $v_{T}$ over the effective particle size as presented in Fig. 4. In order to study only the effects of resonant scattering instead of a combination of $v_{T}$ with the effective refractive index, we have multiplied $v / c$ by $n_{\mathrm{eff}}$, as calculated using ECPA described above. Thus, ideally in the absence of resonance scattering and if ECPA is appropriate, $v_{T}$ is expected to be at a value of 1 , independent of the particle size. As can be seen in the figure, this is the case for some values of the effective diameter, whereas there is a 


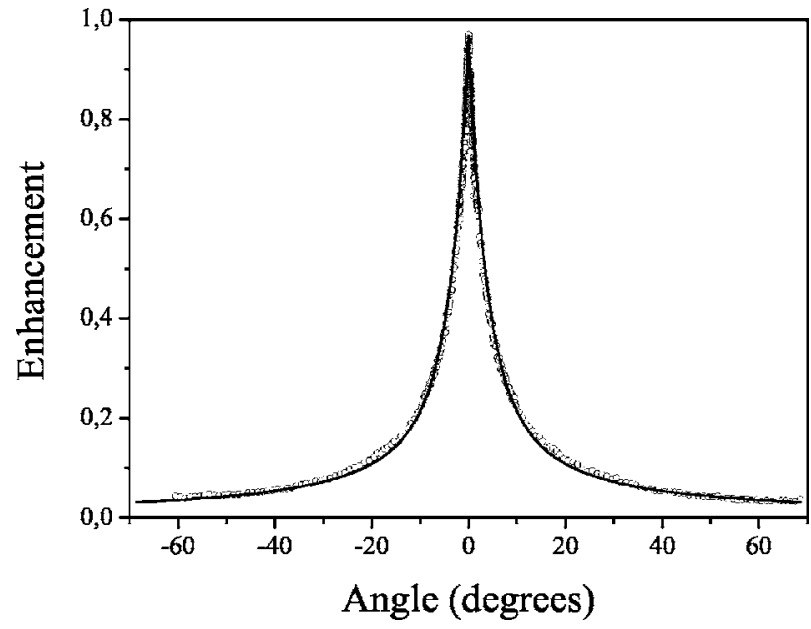

FIG. 3. Angular dependence of the coherent backscattering enhancement from the sample presented in Fig. 2. From the inverse of the full width at half maximum the value of $k l^{*}$ can be determined. Thus a direct measurement of the transport mean free path $l^{*}$ is obtained, since $k$ is the wave number in free space and hence well known. At a wavelength $590 \mathrm{~nm}$, this yields $k l^{*}=6.3$ after taking into account a narrowing due to reflections at the surface [22]. The effective index of refraction has been calculated using ECPA theory [23] in order to be able to correct for the reflectivity.

decrease of the relative $v_{T}$ for effective diameters corresponding to multiples of half the wavelength. This is consistent with a simple geometrical picture of resonant scattering, where the particle diameters have to be such that a standing wave can be excited inside the particle. Due to the polydispersity of the samples of about $20 \%$, we do however, not expect to see sharp peaks for those resonances.

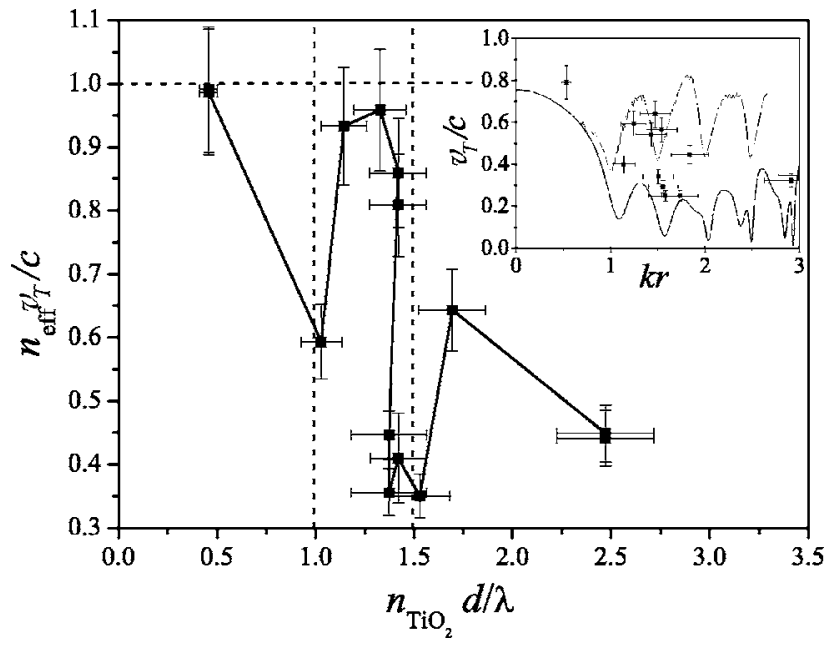

FIG. 4. Measurements of $v_{T}$ normalized to the speed of light in the effective medium as a function of the effective particle diameter. For multiples of half the wavelength, $v_{T}$ shows narrow bands of decreasing values. This is consistent with a simple geometrical picture of resonant scattering. For comparison with theory [8,17] (full line), the same measurements of $v_{T}$, normalized to $c$, are also shown as a function of the size parameter in Mie theory in the inset. The expected behavior of $v_{T}$ from ECPA [24] is also shown as the dashed line for comparison.
The quality of the measurements can be checked by a comparison with the results of an ECPA calculation of the effective refractive index of the matrix medium. It can be seen that for measurements away from a resonance, the value of $n_{\mathrm{eff}} \mathrm{v} / \mathrm{c}$ is close to unity. This shows that the measured value of the effective refractive index is consistent with that calculated using the ECPA approach [23,24].

A comparison of our results with the full theory of van Albada et al. [8] can be seen in the inset of Fig. 4. Here we plot the directly determined $v_{T}$ (without correcting for the effective refractive index) against the size parameter of Mie theory [25], i.e., $x=k r$, where $r$ is the radius of the scatterer. Note that the calculation has been carried out for a refractive index of the particles of 2.73 at a volume fraction of $36 \%$. While our rutile particles have a refractive index between 2.7 and 2.8 at wavelengths of $590 \mathrm{~nm}$, our volume fractions are slightly bigger $(\sim 40 \%)$, which may lead to small shifts. In addition, we compare the data to ECPA theory [24] indicated by the dashed line. As can be seen from the figure, there is good qualitative agreement between our experiments and the theoretical calculations. For instance the first minima of reduction of $v_{T}$ as well as the behavior for bigger particle size are in good agreement with our experiments. However, one can also easily see that quantitatively there are differences of the curves in amplitude. The measured data lie above the theoretical curve of van Albada et al. in the whole range of the investigated effective particle size, while they lie below ECPA inside the resonances. This is probably due to the fact that the theory of van Albada et al. does not take into account correlation effects of the scattering, which may become important at these high packing fractions. For instance it has been shown that positional correlations lead to a significant increase in the value of $l^{*}$ compared to that expected in the independent scattering approximation [26]. Similarly, this treatment of the scatterers as independent Mie spheres can lead to an overestimation of the effective refractive index, which is consistent with the observed discrepancy between theory and experiment. At high filling fractions, ECPA calculations are more appropriate, as is also indicated by our results. For small values of the effective diameter as well as between resonances, the reduction of the energy transport velocity is mostly determined by the effective refractive index. At these particle sizes we find good agreement between the experiment and ECPA as shown above. However, even effects of localization cannot be ruled out at these low values of $l^{*}$ to account for part of the discrepancy between theory and experiment. The samples showing the biggest deviation from theory (around $x=1.25$ ) also show deviations from a classical diffusion pulse which shows the onset of localization in these samples [13].

In conclusion, we have presented direct measurements of the diffusion coefficient and the coherent backscattering cone on various $\mathrm{TiO}_{2}$ samples in order to obtain $v_{T}$ for different particle sizes. For effective diameters corresponding to multiples of half the wavelength, the velocity is strongly reduced by up to a factor of 5. This is in good accord with the occurrence of resonant Mie scattering which should occur when standing waves can be induced inside the samples at exactly these effective diameters. In addition, the same qualitative behavior is found from a full theoretical calculation of the 
transport velocities taking into account resonant scattering. A quantitative agreement in the absolute values of $v_{T}$ between theory and experiment should not be expected due to the fact that theory treats the scatterers as independent Mie spheres [8]. The experimental data away from resonances, however, are more compatible with the results of an ECPA calculation [23] of the refractive index, which takes into account correlation effects. These systematic measurements imply that there is indeed a scatterer dependent reduction of the diffusion constant in multiply scattered light. Observations of anomalously low values of $D[16]$ thus cannot be seen as an indication of Anderson localization.

In earlier experiments, we have observed deviations from the diffusive pulse-shape in strongly scattering samples at long times [13]. This is different from a reduction in transport velocity in that such a reduction only changes the time scale of diffusion on the whole. Localization on the other hand is expected to lead to a scale dependence of $D$, which implies a slower decay of the pulse shape [27,28]. In addition, the observed deviations show a systematic dependence of $l^{*}$ [13], whereas we have shown here that resonant scattering shows a systematic dependence with particle size. Finally, in our experiments, samples with the lowest values of $l^{*}$ do not coincide with effective particle diameters at a resonance. Therefore, this work shows that a separation of the effects of localization and resonant scattering is possible and that the deviations observed in [13] indeed indicate the onset of Anderson localization.

This work was supported by the Deutsche Forschungsgemeinschaft, the International Research and Training Group "Soft Condensed Matter of Model Systems" and the Center for Applied Photonics (CAP) at the University of Konstanz. Furthermore, we would like to thank DuPont chemicals and Aldrich for providing samples used in this study. We would also like to thank Peter Gross for technical support.
[1] E. P. Wigner, Phys. Rev. 98, 145 (1955).

[2] L. V. Hau, Z. Dutton, C. H. Behroozi, and S. E. Harris, Nature (London) 397, 594 (1999); G. Labeyrie, E. Vaujour, C. A. Müller, D. Delande, C. Miniatura, D. Wilkowski, and R. Kaiser, Phys. Rev. Lett. 91, 223904 (2004).

[3] Y. Yamamoto, and R. E. Slusher, Phys. Today 46, 66 (1993); M. L. Gorodetsky, A. A. Savchenkov, and V. S. Ilchenko, Opt. Lett. 21, 453 (1996); J. C. Knight, N. Dubreuil, V. Sandoghdar, J. Hare, V. Lefèvre-Seguin, J.-M. Raimond, and S. Haroche, ibid. 21, 698 (1996).

[4] V. Sandoghdar, F. Treussart, J. Hare, V. Lefèvre-Seguin, J.-M. Raimond, and S. Haroche, Phys. Rev. A 54, R1777 (1996).

[5] F. Vollmer, D. Braun, A. Libchaber, M. Khoshsima, I. Teraoka, and S. Arnold, Appl. Phys. Lett. 80, 4057 (2002).

[6] S. John, Phys. Rev. Lett. 58, 2486 (1987).

[7] Y. Akahane, T. Asano, B. S. Song, and S. Noda, Nature (London) 425, 944 (2003); H. Gersen, T. J. Karle, R. J. P. Engelen, W. Bogaerts, J. P. Korterik, N. F. van Hulst, T. F. Krauss, and L. Kuipers, Phys. Rev. Lett. 94, 073903 (2005).

[8] M. P. van Albada, B. A. van Tiggelen, A. Lagendijk, and A. Tip, Phys. Rev. Lett. 66, 3132 (1991).

[9] P. E Wolf and G. Maret, Phys. Rev. Lett. 55, 2696 (1985).

[10] M. P. VanAlbada and A. Lagendijk, Phys. Rev. Lett. 55, 2692 (1985).

[11] A. F. Ioffe and A. R. Regel, Prog. Semicond. 4, 237 (1960).

[12] P. W. Anderson, Philos. Mag. B 52, 505 (1985).

[13] M. Störzer, P. Gross, C. M. Aegerter, and G. Maret, Phys. Rev.
Lett. 96, 063904 (2006).

[14] E. Abrahams, P. W. Anderson, D. C. Licciardello, and T. V. Ramakrishnan, Phys. Rev. Lett. 42, 673 (1979).

[15] P. W. Anderson, Phys. Rev. 109, 1492 (1958).

[16] J. M. Drake and A. Z. Genack, Phys. Rev. Lett. 63, 259 (1989).

[17] B. A. van Tiggelen, Ph.D. thesis, University of Amsterdam (1992); A. Lagendijk and B. A. van Tiggelen, Phys. Rep. 270, 143 (1996).

[18] R. Lenke and G. Maret, in Scattering in Polymeric and Colloidal Systems, edited by W. Brown and K. Mortensen (Gordon and Breach Scientific, New York, 2000).

[19] G. H. Watson, P. A. Fleury, and S. L. McCall, Phys. Rev. Lett. 58, 945 (1987).

[20] P. Gross, Diploma thesis, University of Konstanz (2005).

[21] E. Akkermans, P. E. Wolf, and R. Maynard, Phys. Rev. Lett. 56, 1471 (1986).

[22] J. X. Zhu, D. J. Pine, and D. A. Weitz, Phys. Rev. A 44, 3948 (1991).

[23] C. M. Soukoulis, S. Datta, and E. N. Economou, Phys. Rev. B 49, 3800 (1994).

[24] K. Busch and C. M. Soukoulis, Phys. Rev. B 54, 893 (1996).

[25] G. Mie, Ann. Phys. 25, 377 (1908).

[26] S. Fraden, and G. Maret, Phys. Rev. Lett. 65, 512 (1990).

[27] R. Berkovits and M. Kaveh, Phys. Rev. B 36, 9322 (1987).

[28] S. E. Skipetrov and B. A. van Tiggelen, Phys. Rev. Lett. 96, 043902 (2006). 